\title{
CMEs and Prominences and Their Evolution Over the Solar Cycle
}

\author{
David F. Webb ${ }^{1}$ \\ Institute for Scientific Research, Boston College, 140 Commonwealth \\ Ave., Chestnut Hill, MA 02467-3862, USA
}

\begin{abstract}
Coronal mass ejections (CMEs) are an important aspect of coronal and interplanetary dynamics. They cause large geomagnetic storms and can drive transient interplanetary shocks, which in turn are a key source of energetic particle events. However, our knowledge of the origins and early development of CMEs at the Sun is limited. CMEs are most frequently associated with erupting prominences and long-enduring soft X-ray arcades, but sometimes with no observed surface activity. I review some of the well determined coronal properties of CMEs and what we know about their source regions, with emphasis on the characteristics of the associated prominences and helmet streamers. One of these characteristics is that many CMEs seem to arise from multipolar magnetic structures with multiple or kinked inversion lines. I also discuss the solar-cycle dependencies of these structures, including the role that erupting prominences and CMEs may play in the ejection of magnetic flux and helicity from the Sun.
\end{abstract}

\section{Introduction}

Coronal mass ejections (CMEs) are now considered a key causal link between solar activity and at least the larger interplanetary (IP) particle events and geomagnetic storms. The onsets of CMEs have been associated with both flares and filament eruptions (e.g., Webb 1992), although many CMEs cannot be associated with any observed surface activity. And, even if accompanied by energetic flares, most of the event energy is contained in the ejected mass and shock wave, not the flare. Most flares occur independently of CMEs and even those accompanying CMEs may be a secondary consequence rather than a cause of CMEs (Gosling 1993). Indeed, the basic physics of the two phenomena may be different, though this assertion has been challenged (e.g., Hudson et al. 1995).

In the next two sections I discuss the origins and characteristics of CMEs at and near the Sun in terms of their basic coronal properties and what we know about their source regions. In section $4 \mathrm{I}$ review some important aspects of the manifestation of CMEs in the inner heliosphere. In section 5 I discuss some solar-cycle dependencies of prominences and CMEs, including the role that erupting prominences and CMEs may play in the ejection of magnetic flux and

\footnotetext{
${ }^{1}$ Also at: Air Force Research Lab./VSBS, Hanscom Air Force Base, MA 01731-3010, USA
} 
helicity from the Sun. I conclude with a summary of the main points.

\section{Basic Properties of CMEs in the Corona}

Most of our understanding of the origins and early development of CMEs has come from analyses of white light observations from spaceborne coronagraphs on the Skylab, P78-1 and SMM satellites viewing within $\sim 10 R_{s}$, from the groundbased Mauna Loa Observatory (MLO) K-coronameter $\left(1.2-2.9 R_{s}\right.$ ), and from the Helios photometers viewing the inner heliosphere from $0.3-1 \mathrm{AU}$. Since early 1996 the SOHO LASCO coronagraphs have been providing unprecedented views of the corona and CMEs from 1.1-30 $R_{s}$ (Howard et al. 1997).

The measured properties of CMEs include their occurrence rates, locations relative to the solar disk, angular widths and speeds (e.g., Webb 1995). Although important quantities, the masses and energies of CMEs require careful instrument calibrations and have large uncertainties. There is a large range in the basic properties of CMEs. Their speeds, accelerations, masses and energies extend over 2-3 orders of magnitude, and their widths exceed by factors of 3-10 the sizes of flares and active regions.

The basic structure of many CMEs appears to consist of three main components: a bright leading arc followed by a dark, low-density cavity and a bright core of denser material. Bright cores were observed in about $1 / 4$ of the SMM CMEs. One view is that the pre-event structures which erupt to become the CME consist of a prominence, its overlying coronal cavity, and the ambient corona. Figure 1 shows a classic example of a "3-part" CME; as shown here the prominence itself constitutes a small, but dense fraction of the entire CME volume (Gopalswamy et al., these proceedings). The CME "loop" may actually be the skyplane projection of a 3-D, shell-like structure. It must be emphasized that CMEs exhibit a variety of forms, some having complex structure with their interiors filled with bright emitting material. LASCO observations suggest that many CMEs are accompanied by a global response of the corona, especially along the streamer belt.

Sufficient data on CMEs have been obtained to permit study of their frequency of occurrence over about 1.5 solar cycles (Webb and Howard 1994). Data from the earlier coronagraphs and the Helios photometers were corrected for duty cycles and visibility effects to study the annual CME rate. The CME rate tracks the solar cycle in both phase and amplitude, which varies by an order of magnitude over the cycle. The more sensitive LASCO observations indicate that CMEs may be even more frequent, since the CME rate in 1996 was a factor of 2-3 higher than during the previous solar minimum (Howard et al. 1997).

The latitude distribution of the central position angles of SMM CMEs was very broad near cycle maximum but clustered about the equator at minimum. In contrast, the size distribution (i.e., angular widths) of SMM CMEs did not vary much over the cycle, maintaining an average width of about $45^{\circ}$ (Hundhausen 1993). However, the SOLWIND CME size distribution did change between cycle maximum and minimum, with the average width decreasing from 45 to $24^{\circ}$ (Howard et al. 1985). Thus far, the LASCO CME widths have averaged 45-50 $0^{\circ}$, but more wider, halo-type CMEs have been observed.

Estimates of the speeds of the leading edges of CMEs range from about 


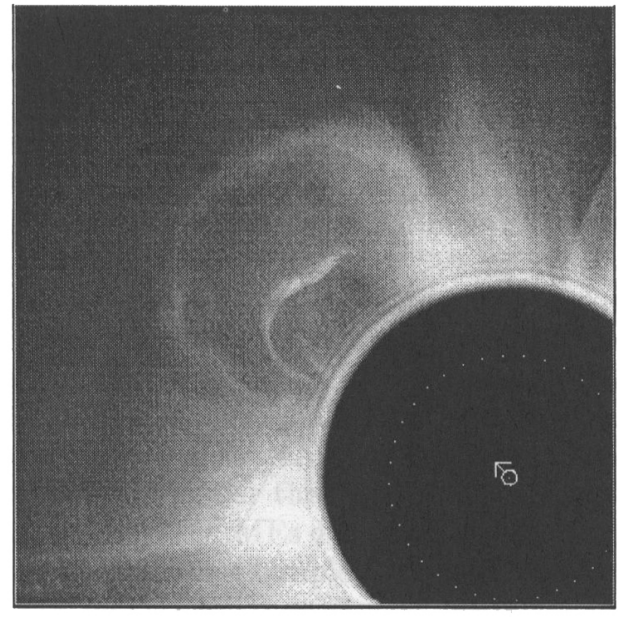

Figure 1. A CME observed from SMM on April 14, 1980. The event shows the "3-part" structure of a CME: an outer, bright loop followed by a darker cavity and bright inner core, here an erupting prominence viewed side on. Courtesy High Altitude Observatory/NCAR.

20 to $2000 \mathrm{~km} \mathrm{~s}^{-1}$. The annual average speeds of SMM CMEs varied over the solar cycle from $160-460 \mathrm{~km} \mathrm{~s}^{-1}$, but were not simply related to sunspot number (Hundhausen et al. 1994). The speed of a CME is independent of its heliolatitude. CMEs exhibit a differential speed gradient, having a smooth gradient of speeds from the fast leading edge to the lower, interior material, such as that of the prominence. Higher speed $\left(>400 \mathrm{~km} \mathrm{~s}^{-1}\right)$ CMEs tend to be associated with coronal and IP shocks, suggesting that the shocks are piston-driven. At least through the lower corona, typical CMEs appear to travel at constant velocity, although some events exhibit significant acceleration. Acceleration has been observed in many LASCO CMEs out to $30 R_{s}$.

The average coronagraph values for CMEs are masses of a few $\times 10^{15} \mathrm{~g}$ and energies of about $3 \times 10^{30} \mathrm{erg}$. However, the average CME masses and kinetic energies derived from Helios photometer data in the interplanetary medium are 2-8 times higher than those determined by coronagraphs (Jackson et al. 1996). With the greater field of view of LASCO, CME mass estimates increase as the CME front moves outward, suggesting continual outward flow (Howard. et al. 1997). This implies that previous coronagraph masses have been underestimated by as much as a factor of ten. The energy spectrum (event rates vs energies) of CMEs does not follow the same simple power law distribution as that of $\mathrm{X}$-ray flares (Figure 2). In addition, SMM CME masses were not distributed like a power law, having a sharp cutoff at $10^{-13} \mathrm{~g} / \mathrm{deg}$. Along with other evidence presented here, this suggests that flares and CMEs arise in different physical processes. However, LASCO results reveal many small events travelling through streamers that may refine this distribution. But are these all CMEs? 


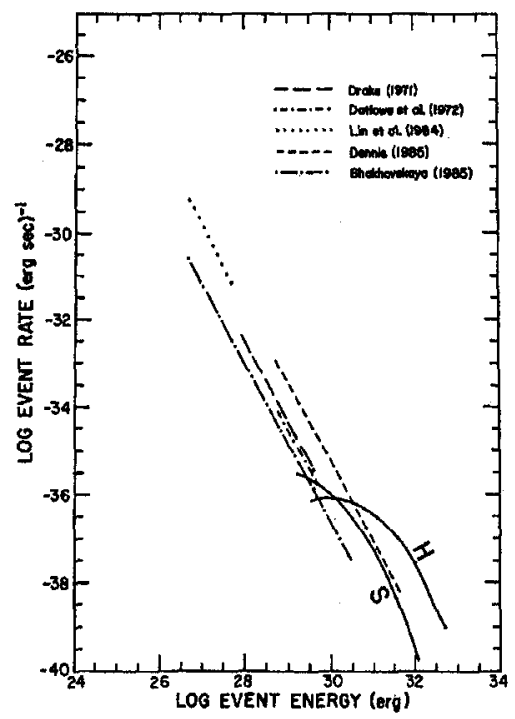

Figure 2. Comparison of solar X-ray flare energy vs. peak flux measurements with CME kinetic energy distributions from Solwind (S) and Helios (H). From Jackson (1996).

\section{Source Regions of CMEs}

From what kind of structures do CMEs arise? Recent studies suggest that CMEs arise from large-scale, closed structures, most of which are preexisting coronal streamers (Hundhausen 1993). Although the solar origins of CMEs remain obscure, they are often associated with the disruption of large-scale structures such as prominences and loop arcades. Low and Hundhausen (1995) emphasize that the prominence/streamer involves a dual flux system, one part of which is a flux rope which, with its surrounding coronal cavity, may drive the eruption once the streamer is disrupted. CMEs seem to be more closely linked to large-scale weaker magnetic structures than to small-scale stronger-field regions. Many energetic CMEs are actually the disruption ("blowout") of a preexisting streamer, which increases in brightness and size for days before erupting as a CME. Afterwards the streamer and CME are absent, giving the appearance of a "bugle" on white light synoptic maps (Hundhausen 1993). The streamer usually reforms a day or so later and sometimes a bright, narrow ray appears above it, likely tracing the current sheet.

Statistical association studies typically indicate that CMEs are most frequently associated with near-surface activity in the form of erupting filaments and X-ray long duration events, but not optical flares. However, the fastest, most energetic CMEs are usually flare-associated (see Webb 1992 for a review). In these studies a third to a half of all CMEs cannot be associated with any nearsurface activity. The bright cores of material within many CMEs also suggest that erupting prominences are more fundamentally involved in the initiation of CMEs than are flares.

We now review results on the source regions of CMEs using soft X-ray and 
white light data sets from two different decades; the 1980s and 1990s. During the 1980s there was excellent coverage of white light CMEs from the Solwind and SMM coronagraphs and the HAO MLO K-coronameter. However, X-ray data consisted mostly of whole-Sun GOES X-ray data. Comparing the Solwind CME and GOES X-ray data, Sheeley et al. (1983) found that the probability of associating a CME with a soft X-ray flare increased linearly with the flare duration, reaching $100 \%$ for flares of duration $\geq 6 \mathrm{hr}$.

The SMM CME observations indicated that the departure times of flareassociated CMEs typically preceded the flare onsets. Harrison (1991) suggested a model in which CMEs were initiated during weaker "precursor" soft X-ray bursts that preceded any subsequent main flare by several to tens of min. These precursor bursts consisted of X-ray arches having large scale sizes of $10^{5} \mathrm{~km}$. The flares could lie anywhere under the span of the accompanying CME.

The characteristics of SMM CMEs did not seem well correlated with those of any associated X-ray events. For example, Hundhausen (1997) shows examples of two CMEs in 1989, both similar with fast leading loops and cavities and associated with the eruption of prominences extending onto the disk. In each the CME had formed and was moving outward before the onset of any signature in GOES X-rays. However, one CME was associated with a bright M3 longduration X-ray event, while with the other CME there was no discernable X-ray event above the background. Hundhausen draws three conclusions from his studies: 1) Intense X-ray flares are neither a necessary nor sufficient condition for the occurrence of CMEs; 2) Any significant X-ray emission follows the onset of the CME and peaks much later; and 3) The intensity of any soft X-ray event accompanying a CME is not well related to the characteristics of the CME.

During the present decade of the 1990s, a clearer understanding of CME onset structures has been gained using the temporally and spatially resolved Yohkoh SXT soft X-ray data. However, between late 1989 when SMM ended and early 1996 when SOHO became operational, we had only limited white light CME coverage provided by the MLO K-coronameter. Despite this limited CME data, the Yohkoh data is helping us gain insight into the source regions of CMEs. Klimchuk et al. (1994) identified $\sim 30$ eruptive X-ray loop events occurring during MLO observing periods in the SXT images at the limb. They concluded that the speeds, widths, and occurrence rates of these loops were consistent with the early phase of CMEs. Hundhausen and colleagues and Hudson and Webb have directly compared MLO CMEs with the SXT images. Hudson and Webb (1997) found that $2 / 3$ of the CMEs had an associated transient X-ray structure, typically a loop with one foot in a flaring active region. Hundhausen (1998) summarized the HAO results and gave examples for CMEs at low heliolatitudes in the active region belt. The CME blows out a typically $40^{\circ}$ wide structure at $2 R_{s}$. If an $\mathrm{X}$-ray event is associated, it will be intense but of smaller scale and offset toward one leg of the CME. At higher latitudes the associated X-ray events are of larger scale and longer duration but tend to have weaker emission. Since many characteristics of CMEs appear independent of latitude, we can conclude that CMEs involve the destabilization of large-scale coronal structures which result in magnetic reconnection of the larger-scale, weaker fields at higher latitudes and of the smaller-scale, stronger fields at low latitudes.

Hansen et al. (1974) and Rust and Hildner (1976) noted the depletion of 
lower coronal material during eruptive events in the Skylab era. Now in the SXT data areas of subtle dimming, evidence of density depletion, have been observed above the brightening $\mathrm{X}$-ray arcade regions (reviewed by Hudson and Webb 1997; see Gopalswamy et al. and Hiei, these proceedings). Two outstanding examples, on 21 February and 28 August 1992, may provide views along and across the reconnecting arcades (Hudson et al. 1996; Watanabe et al., these proceedings). One interpretation of the dimming signature is that the initially closed field lines are opening during the onset of a CME, in analogy to transient coronal holes observed against the disk. Therefore, such dimming may be one of the first soft X-ray signatures of the mass ejection in the low corona.

Some observations suggest that multiple polarity systems may be involved in many CMEs. The widths of CMEs $\left(\sim 50^{\circ}\right)$ are large compared with the scale of surface polarity inversion lines (separations of $10-20^{\circ}$ ). A number of total solar eclipse observations reveal this disparity, showing single streamers overlying twin coronal arcades and parallel filament segments, for instance during the 1966 eclipse. The eruption of such a helmet streamer would then suggest that the source region of the resulting $\mathrm{CME}$ consisted of a complex magnetic system of multiple arcades and neutral lines. Webb et al. (1997) find that transient X-ray arcades, used as proxies for CMEs against the disk, can span surface distances of 35 to $>100^{\circ}$ and cross $2-3$ neutral lines or a single, highly convoluted neutral line, implying multipolar magnetic systems. In a separate study we also find that CMEs tended to occur over surface sites where opposite polarity regions were rapidly evolving. Observations and models involving quadrupolar fields supporting prominences and their eruption as CMEs were much in display during this workshop (e.g., papers by Uchida, Demoulin, and Cheng and Choe in these proceedings). The recent LASCO data also support this picture in that the single equatorial streamer belt tends to overlie quadrupolar structures at lower heights (e.g., Howard et al. 1997). This supports the model of Crooker et al. (1993) that the streamer belt, the base of the heliospheric current sheet, can be broad and contain multiple current sheets. Thus, the current sheet acts as a conduit for CMEs and is one reason why transient interplanetary flows in the ecliptic are often associated with stream-stream interaction regions.

\section{Interplanetary Signatures of CMEs}

CMEs carry into the heliosphere large amounts of coronal magnetic fields and plasma, which can be detected by remote sensing and in-situ spacecraft observations. CME plasma has been remotely detected by the twin Helios photometers and with limited success by ground-based measurements of IP scintillation. The Helios photometers detected about $200 \mathrm{CMEs}$ over a period of 9 years (Jackson et al. 1994). The morphology, occurrence rates, spans and speeds of these CMEs are consistent with coronagraph measurements made near the Sun (Webb and Howard 1994).

A variety of in-situ signatures have been proposed as proxies for the passage of CME ejecta past spacecraft. These include IP shocks, density changes, decreased temperatures, flows with enhanced helium abundances, and magnetic field structures consistent with looplike topologies (Gosling 1993). Recent efforts have focussed on signatures indicative of the topology of the ejected magnetic 
fields, such as magnetic clouds and bidirectional particle flows.

Bothmer and Schwenn (1994), Rust (1994), Bothmer and Rust (1997) and others have associated some magnetic clouds with solar filament disappearances. The close association of CMEs with filament eruptions and shearing fields near the surface has led to the modeling of CMEs as flux ropes. Gosling (1993) suggested that $\sim 1 / 3$ of all bidirectional electron events and magnetic clouds in the heliosphere have the characteristics of flux ropes. Marubashi (1997) and Bothmer and Schwenn found that the IP flux ropes (clouds) they examined had the same orientation and polarity as associated erupting solar filaments. Furthermore, Martin (these proceedings) found that high latitude filaments always have twist in the same sense in a given hemisphere. These results suggest that the sign of the helicity, or twist of the erupting fields can be predicted assuming an association between a given flux rope and a filament eruption. Rust (1994) did so for a list of magnetic clouds at $1 \mathrm{AU}$ and claimed good agreement. Thus, filament eruptions and CMEs may be important ways that the Sun sheds helicity.

Recently, LASCO has observed a number of halo-type CMEs, which had been rarely observed by the earlier less-sensitive coronagraphs. A halo-type CME, especially when associated with solar activity near sun center, suggests the launch of a geoeffective disturbance toward Earth. Indeed, many of the recent halo CMEs have been associated with magnetic clouds and geomagnetic storms at Earth $\leq 4$ days later. Several of these events were associated with filament eruptions and X-ray arcades at the Sun (e.g., Gopalswamy et al., Plunkett et al., and van Driel-Gesztelyi et al., these proceedings) followed by in-situ observations from the WIND and SOHO spacecraft at $1 \mathrm{AU}$ providing evidence of cool prominence material at the trailing edge of a magnetic cloud.

Figure 3 shows one such event observed by WIND in January 1997. A halo $\mathrm{CME}$ and small, sun-centered disappearing filament on January 6 (Webb et al. 1998) was followed on January 10-11 by a magnetic cloud which drove a geomagnetic storm. An unusually dense "plug" of material at the back of the cloud exhibited an enhanced helium abundance, cool $\mathrm{He}^{+}$material, and charge states typical of a large range of temperatures. Such unusual solar wind conditions have always been interpreted as evidence for solar filament material encased in transient CME flows. Burlaga et al. (1998) have modeled the magnetic cloud as a force-free flux rope with its axis nearly parallel to the ecliptic. The cloud contained intervals of bidirectional electron flows indicative of closed, transient fields, and ended at an interface with a corotating high speed stream.

\section{Solar-Cycle Variation of Prominences and CMEs}

The temporal and latitudinal distributions of streamers and prominences are similar to those of CMEs, being confined to low latitudes about the current sheet near solar cycle minimum and becoming broadly distributed near maximum (Hundhausen 1993). This evolution is different from that of active regions, flares or sunspots. The impression is that CMEs are more closely linked to large-scale weaker magnetic structures than to small-scale stronger-field regions.

Filaments exhibit their own large-scale "butterfly diagram" on latitudetime plots with two distinct branches "flowing" toward the poles and toward the 


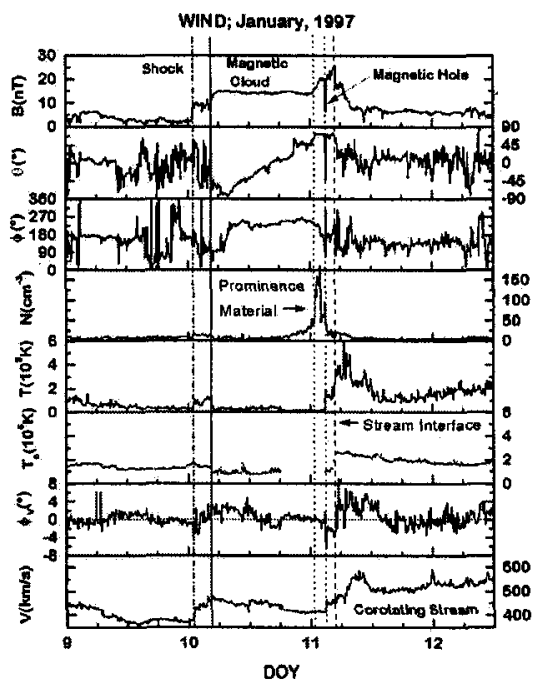

Figure 3. Solar wind plasma and magnetic field data from the WIND spacecraft at Earth in January 1997. A shock on Jan. 10, 0100 UT (dashed line) was followed by a classic magnetic cloud (onset at solid line), a dense "plug" of material (filament), and a corotating stream. From Burlaga et al. (1998).

equator (Minarovjech et al., these proceedings). The higher-latitude filaments display the following characteristic pattern over each solar cycle: a "rush to the poles" and development of a lower crown during the cycle rise, disappearance of the polemost, or "true" crown during solar maximum and the polarity reversal, and subsequent dwelling at $\sim 50^{\circ}$ during the fall of the cycle and minimum of the new polar crown. McIntosh (1992) noted that high-latitude filaments fall into two groups, those having "correct" polarity for the cycle in that hemisphere, i.e., the magnetic field lying poleward of the polar filaments will be appropriate for that cycle, and those having opposite polarity. For cycles 20-22, McIntosh determined the maximum latitude, north and south of the equator for each rotation, for both types of filaments. His result is shown in Figure 4.

Cliver and colleagues have recently published two studies comparing this pattern of filament evolution with CMEs. Cliver et al. (1994) showed that the CME rate during cycle 22 exhibited a quasi-discontinuity in October 1988, when the average daily rate doubled and remained high until the end of SMM observations in late 1989. This jump was most pronounced in the population of high-latitude CMEs. They inferred a similar discontinuity in the CME rate at the same time of the previous cycle in 1978. These discontinuities are marked on Figure 4 by vertical arrows; they occur at about the time of the start of the poleward movement of the two filament crowns. Cliver and Webb (these proceedings) take the next step by statistically comparing high-latitude CMEs with disappearing solar filaments (DSFs). They find that the fraction of DSFs arising from the true polar crown decrease until solar maximum when the new, or emerging polar crown becomes the dominant source of high-latitude DSFs, 


\section{Polar Crown Filaments Cycles 20-21-22}

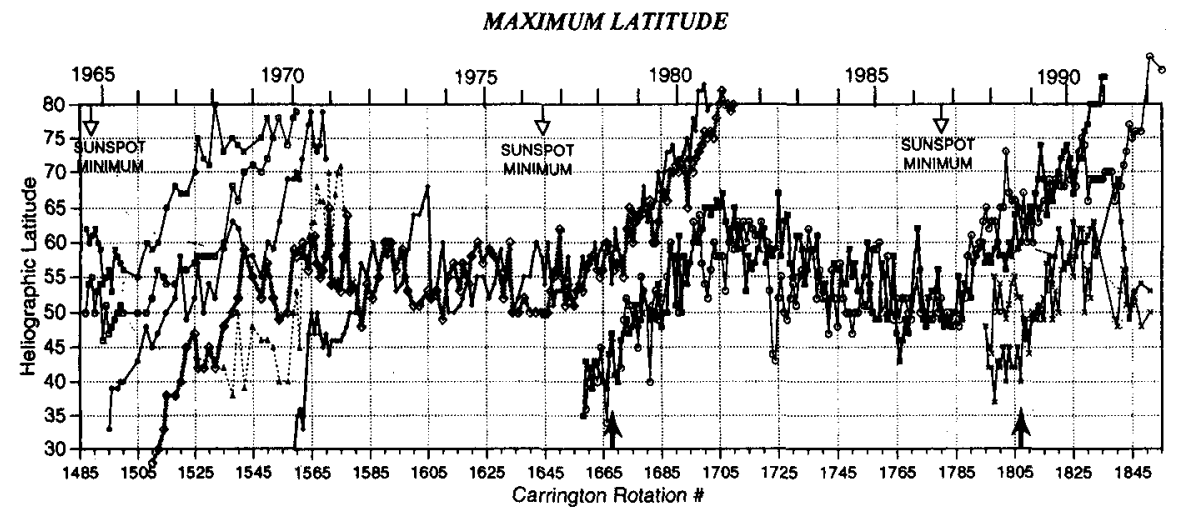

Figure 4. The maximum latitude of polar crown filaments in the true and emergent polar crowns during cycles $20-22$. Filament positions are given separately for the northern and southern hemispheres. Arrows at bottom mark the epochs in cycles 20 and 21 when the CME rate abruptly increased. (Figure courtesy P. S. McIntosh.)

and by inference of high-latitude CMEs. However, at the maxima of the last two solar cycles, there were $\sim 4$ times as many high-latitude CMEs as high-latitude DSFs. Possible reasons for this discrepancy are discussed by Cliver and Webb. A related phenomena that has been discovered is that the coronal structures overlying the polar crown regions appear to be hotter than other non-active regions areas in the corona. Guthathakurta et al. (1993), Hick et al. (1996) and others have used Sacramento Peak Observatory Fe-line intensity ratios to calculate large-scale temperatures at several heights in the corona. They find that there are distinct high-latitude $\left(>50^{\circ}\right)$ bands of coronal emission which exist over most of the cycle. These bands correspond to high-latitude white light streamers and the current sheet, and are hotter than elsewhere. In a gross sense these high temperature bands overlie the polar crown and follow its poleward movement during the cycle. It is interesting to speculate whether or how these bands are related to the occurrence of high-latitude DSFs and CMEs.

There are important new results concerning the interrelationships among solar filaments and the global helicity balance, and how that helicity builds up during the cycle and is ejected from the Sun. As mentioned earlier, several studies find that magnetic clouds modeled as flux ropes have the same orientation and polarity as associated erupting filaments at the sun. Since higher latitude filaments in a given hemisphere have the same helicity (twist) and the sign of the helicity of DSFs associated with clouds at $1 \mathrm{AU}$ agree, filament eruptions and CMEs seem to be important ways that the Sun can shed the helicity that is built up over a cycle (Seehafer, these proceedings).

Recently, Bothmer and Rust (1997) found a dependence of cloud magnetic 
structure on the solar cycle phase. During cycle 20-21 (1974-1981) most magnetic clouds at $1 \mathrm{AU}$ exhibited a southward then northward (SN) field rotation, whereas during cycle 21-22 (1982-1991) clouds with NS rotation dominated. Based on these results, an empirical model from Bothmer and Schwenn (1994), and the helicity rule of Rust and Kumar (1994), Bothmer and Rust show that both the occurrence and rotation of clouds might be predictable. For example, the majority of clouds during the next few years should be SN and, therefore, associated geomagnetic storms may be more intense because SN clouds are more geoeffective. Supporting this, the recent January 1997 storm was caused by a SN cloud (Figure 3). In addition, Rust and Kumar (1994), Martin and McAllister (1997) and others have identified patterns of soft X-ray sigmoid structures and the skew of arcades with filaments and DSFs in each hemisphere. Together, these results suggest that studies relating the solar cycle dependencies of filaments, DSFs, CMEs and magnetic clouds will help us to understand the build up and ejection of magnetic flux and helicity from the Sun.

\section{Conclusion}

I have reviewed the origins and characteristics of CMEs at the Sun in terms of their basic coronal properties and what we know about their source regions, and some important aspects of their manifestation in the inner heliosphere. The rate of occurrence of CMEs tracks solar activity, but the size scales of CMEs are much larger than and their latitude distributions different than those of near-surface activity like flares or active regions. Most CMEs arise in large-scale closed coronal structures, especially helmet streamers which erupt and reform. Statistically, CMEs are most frequently associated with erupting filaments and $\mathrm{X}$-ray long duration events, not optical flares. However, the Yohkoh data is helping to reshape our ideas on these associations. Large-scale X-ray arcades are frequently observed, and likely result from reconnection of closed field systems opened by CMEs. The size scales and field strengths of these systems are a function of latitude; the arcades are larger-scale but weaker at higher latitudes and smaller-scale and stronger at low latitudes. The magnetic structures involved with the sources of CMEs can be complex and multipolar. The earliest $\mathrm{X}$-ray signatures of the onset of a CME in the low corona appear to include outward-moving loops and the depletion of coronal material above the bright $\mathrm{X}$-ray arcade. These observations and comparison of the energy distributions of $\mathrm{X}$-ray flares and CMEs suggest that the two phenomena may arise in different physical processes.

In terms of solar-terrestrial linkages, CMEs are now identified as a crucial link between solar eruptions and their propagation through the heliosphere to Earth. The IP manifestations of CMEs can result in extensive transient disturbances and can cause major geomagnetic storms at Earth. CMEs are associated with the coronal streamer belt and, therefore, with the heliospheric current sheet. Recent studies show that many magnetic clouds arise as solar filament flux ropes. The magnetic helicity or twist of filaments and DSFs follows specific patterns in time and location, and has been related to the magnetic orientation of clouds. This suggests that DSFs and CMEs may be important ways that the Sun sheds its helicity built up over the cycle. Halo CMEs and associated near Sun-center 
DSFs are followed by clouds at Earth, permitting in-situ measurements of the internal CME structure including any filament material.

Acknowledgments. I thank E. Cliver and S. Kahler for helpful comments and the SOC and LOC for the invitation and financial support. Some of this work was supported at Boston College by the Geophysics Directorate of the USAF Phillips Lab (now AF Research Lab) under contract AF19628-96-K-0030.

\section{References}

Bothmer, V. and R. Schwenn 1994, in Mass Supply and Flows in the Solar Corona, (eds.) B. Fleck et al., Kluwer, Norwell, MA, p. 225

Bothmer, V. and D. M. Rust 1997, in Coronal Mass Ejections, (eds.) N. Crooker et al., GM 99, AGU, Washington, DC, p. 139

Burlaga, L. et al. 1998, J. Geophys. Res., 103, 277

Cliver, E. W., O. C. St. Cyr, R. A. Howard and P. S. McIntosh 1994, Solar Coronal Structures, (eds.) V. Rusin et al., Veda, Bratislava, p. 83

Crooker, N. U., G. L. Siscoe, S. Shodan, D. F. Webb, J. T. Gosling, and E. J. Smith 1993, J. Geophys. Res., 98, 9371

Gosling, J. T. 1993, J. Geophys. Res. 98, 18,937

Guhathakurta, M., R. R. Fisher and R. C. Altrock 1993, ApJ, 414, L145

Hansen, R. T., C. J. Garcia, S. F. Hansen, and E. Yasukawa 1974, PASP, 86, 500

Harrison, R. A. 1991, Adv. Space Res., 11 (1), 25

Hick, P., B. V. Jackson and R. C. Altrock 1996, in Solar Wind Eight, (eds.) Winterhalter et al., AIP, Woodbury, NY, p. 169

Howard, R. A. and 18 coauthors 1997, in Coronal Mass Ejections, (eds.) N. Crooker et al., GM 99, AGU, Washington, DC, p. 17

Howard, R. A., N. R. Sheeley, M. J. Koomen and D. J. Michels 1985, J. Geophys. Res., 90,8173

Hudson, H. S. and D. F. Webb 1997, in Coronal Mass Ejections, (eds.) N. Crooker et al., GM 99, AGU, Washington, DC, p. 27

Hudson, H., B. Haisch, and K. T. Strong 1995, J. Geophys. Res. 100, 3473

Hudson, H. S., J. R. Lemen and D. F. Webb 1996, in Magnetic Reconnection in the Solar Atmosphere, (eds.) R. D. Bentley and J. T. Mariska, ASP Conf. Ser. Vol. 111, San Francisco, p. 379

Hundhausen, A. J. 1993, J. Geophys. Res. 98, 13,177

Hundhausen, A. J., 1997, in Cosmic Winds and the Heliosphere, (eds.) J. Jokipi et al., University of Arizona Press, Tucson, AZ

Hundhausen, A. J. 1998, in The Many Faces of the Sun, (eds.) K. Strong et al., Springler-Verlag, New York, in press

Hundhausen A. J., J. T. Burkepile and O. C. St. Cyr 1994, J. Geophys. Res., 99,6543

Jackson, B. V. 1996, in Magnetic Storms, (eds.) B. Tsurutani et al., GM 98, AGU, Washington, DC, p. 59 
Jackson, B. V., R. A. Howard and D. F. Webb 1996, Solar Wind Eight, (eds.) Winterhalter et al., AIP, Woodbury, NY, p. 540

Jackson, B. V., D. F. Webb, P. L. Hick, and J. L. Nelson 1994, Catalog of Helios 90 $0^{\circ}$ Photometer Events, PL-TR-94-2040, Phillips Laboratory, Hanscom $\mathrm{AFB}, \mathrm{MA}$

Klimchuk, J., L. Acton, K. Harvey, H. Hudson, K. Kluge, D. Sime, K. Strong, and T. Watanabe 1994, in X-ray Solar Physics from Yohkoh, (eds.) Y. Uchida et al., Universal Academy Press, Tokyo, p. 181

Low, B. C. and J. R. Hundhausen 1995, ApJ, 443, 818

Martin, S. F. and A. H. McAllister 1997, in Coronal Mass Ejections, (eds.) N. Crooker et al., GM 99, AGU, Washington, DC, p. 127

Marubashi, K. 1997, in Coronal Mass Ejections, (eds.) N. Crooker et al., GM 99, AGU, Washington, DC, p. 147

McIntosh, P. S. 1992, in The Solar Cycle, (ed.) K. Harvey, ASP Conf. Ser. Vol. 27, San Francisco, p. 14

Rust, D. M. 1994, Geophys. Res. Lett. 21, 241

Rust, D. M. and E. Hildner 1976, Solar Phys., 48, 381

Rust, D. M. and A. Kumar 1994, Solar Phys., 155, 69

Sheeley, N. R., R. A. Howard, M. J. Koomen and D. J. Michels 1983, ApJ, 272, 349

Webb, D. F. 1995, Rev. Geophys. Suppl., 577

Webb, D. F. 1992, in Eruptive Solar Flares, (eds.) Z. Svestka, B. Jackson and M. Machado, Springer-Verlag, New York, p. 234

Webb, D. F. and R. A. Howard 1994, J. Geophys. Res. 99, 4201

Webb, D. F., S. W. Kahler, P. S. McIntosh and J. A. Klimchuk 1997, J. Geophys. Res., 102, 24,161

Webb, D. F., E. W. Cliver, N. Gopalswamy, H. S. Hudson and O. C. St. Cyr 1998, Geophys. Res. Lett., in press 